\section{El derecho a la salud en Colombia: una propuesta para su fundamentación moral}

\author{
Luz Stella Álvarez Castaño ${ }^{1}$
}

Palabras clave: justicia social, prestación de atención de salud, recursos en salud, asignación de recursos, derechos humanos, reforma en atención de la salud, Colombia.

1 Universidad de Antioquia, Antioquia, Colombia. La correspondencia debe dirigirse a carrera 75 No 65-87, Medellín, Colombia. Correo electrónico: lalvar9@uic.edu
En Colombia se implantó en 1993 una reforma del sistema de salud fundamentada moralmente en la doctrina liberal y orientada a incrementar los mecanismos del mercado en el sector de la salud (1). Aunque la reforma se promovió como un mecanismo necesario para resolver los problemas del sector y especialmente para atenuar las inequidades que golpeaban con mayor fuerza a los más pobres, esta promesa no se cumplió y algunas inequidades incluso se acentuaron.

Sectores académicos, organizaciones sociales y parte de la sociedad política han postulado el enfoque del derecho a la salud como alternativa al modelo implantado en 1993. Aunque la fuerza de este enfoque en el plano social y su capacidad para articular parte de los movimientos sociales relacionados con la salud han crecido, sus fundamentos teóricos no han tenido el mismo desarrollo. Por ejemplo, en Colombia el derecho a la salud tiene antecedentes en el campo jurídico-legal, pero su fundamentación en el plano ético-moral es más reciente.

La fundamentación jurídico-legal de un derecho está compuesta por el conjunto de normas y leyes que lo garantizan, establecidos por los mecanismos e instancias encargados de su cumplimiento. En el caso colombiano, el derecho a la salud está fundamentado jurídica y legalmente en algunos compromisos internacionales firmados por el Estado (2). Por su parte, la fundamentación moral de un derecho es el conjunto de principios éticos que lo sustentan como derecho fundamental, independientemente de que exista o no una ley que lo respalde.

El objetivo de este artículo es contribuir a la fundamentación moral del derecho a la salud en Colombia, tomando como referencia algunas de las teorías de justicia social desarrolladas en el campo de la ética y la filosofía moral.

La reforma del sistema de salud colombiano: el enfoque neoliberal-utilitarista

Una de las reformas más emblemáticas en América Latina es la que se aplicó al sistema de salud colombiano, el cual, después de reformado, fue catalogado como el más equitativo del mundo por su desempeño financiero (3). El sistema anterior estaba compuesto por tres subsistemas:

- el subsistema público, conformado por una red de hospitales y centros de salud que atendía a las 
personas de bajos ingresos y a los desempleados y estaba financiado con fondos que otorgaba el Estado directamente a las instituciones prestadoras de servicios;

- el subsistema de aseguramiento, conformado por una red de hospitales y centros para la atención de los trabajadores, financiado con aportes de los empleadores y empleados;

- el subsistema privado, formado por instituciones que atendían a quienes pagaban la atención de forma directa o mediante sistemas de prepago (15\% de la población) (4).

La reforma colombiana se basó en los conceptos del pluralismo estructurado (5) y de la llamada atención controlada o managed care $^{2}$. En términos generales, los lineamientos que siguió el modelo aplicado fueron: a) implantación del sistema de aseguramiento, b) creación de un mecanismo de mediación financiera entre los asegurados y los proveedores de los servicios, que introdujo en el sector de la salud a empresas aseguradoras del sector financiero, c) establecimiento de un sistema de subsidio parcial para las personas sin capacidad de pago, y d) aplicación de un plan básico de beneficios en el que los pacientes debían cubrir las necesidades no contempladas por el plan.

El modelo aplicado en Colombia se fundamentó moralmente en la teoría liberal, que privilegia el respeto a los derechos civiles y políticos denominados "negativos" (a la vida, la integridad física, la libertad, la propiedad privada y el voto). Estos derechos existen para todas las personas aunque el Estado no los haya transformado en derechos "positi$\operatorname{vos}^{\prime \prime}$, es decir, no los haya reconocido mediante leyes (6). Como la teoría liberal considera fundamentales solo estos derechos, son los únicos que los ciudadanos le pueden exigir al Estado. Según la doctrina del liberalismo, la asistencia sanitaria es una prerrogativa social que puede ser solicitada en virtud del principio de beneficencia, pero no exigida (6). Según el pensamiento liberal, los derechos civiles y políticos son de obligación perfecta, es decir, generan deberes para los demás, mientras que las prerrogativas sociales son de obligación imperfecta, pues no generan la obligación del Estado o de los demás miembros de la sociedad. Las personas pueden ejercer los principios de caridad o beneficencia de manera voluntaria y cuando así lo deseen (6). Por tanto, la noción de justicia sanitaria según el pensamiento liberal es que existe un derecho negativo a la salud, entendido esto como el derecho de las perso-

\footnotetext{
Aunque no hay una definición precisa, este término se usa generalmente para designar un grupo amplio de prácticas usadas para la contratación y provisión de servicios de salud de manera corporativa (negociaciones entre proveedores, aseguradores, empleadores) con el objetivo aparente de disminuir costos en el sector salud.
}

nas a exigir que su vida e integridad física sean respetadas, pero no como un derecho positivo a la asistencia sanitaria, es decir al cuidado de la salud. En otras palabras, según el liberalismo, el cuidado de la salud no es un derecho humano fundamental (6).

No existe una corriente única de pensamiento liberal. Hay diferentes enfoques dentro de esa escuela y un mismo término se usa para designar posturas filosóficas y políticas diferentes en dependencia del país. Por lo tanto, es necesario precisar que en este texto se entenderá por enfoque liberal o neoliberal la versión más ortodoxa del liberalismo, cuyos preceptos básicos se mencionan en el párrafo anterior.

En términos generales, los sistemas de salud en los que se aplica la doctrina liberal se organizan alrededor de dos grandes pilares:

a) los servicios curativos, que se someten a las leyes del mercado y generan una asistencia sanitaria diferenciada, en la que cada persona recibe la atención de salud que puede pagar;

b) la salud pública, que se deja en manos del Estado, pues aunque es valorada por la sociedad como muy importante, no es rentable económicamente.

En el caso colombiano, el ideario neoliberal no llegó solo, pues vino acompañado de la retórica y de la aplicación de mecanismos utilitaristas. El utilitarismo determina el valor de los programas de salud y de las estrategias sanitarias según la relación entre los resultados obtenidos y el dinero invertido. La concepción utilitarista se utilizó como herramienta de contención de los costos y fue presentada como un conjunto de indicadores que permitían mejorar la asignación de los recursos para la salud, de acuerdo con la importancia relativa de las diferentes enfermedades o grupos de enfermedades, la carga de enfermedad en diferentes poblaciones y los beneficios ganados en las diferentes intervenciones de salud (7).

La aplicación del paradigma neoliberalutilitarista en Colombia provocó una mayor inequidad en los servicios de salud. Según Whitehead, las inequidades en materia de salud son "diferencias innecesarias y evitables, que además se consideran injustas" (8). En materia de cobertura, no es posible analizar comparativamente los cambios provocados por la nueva política con respecto a la situación anterior, ya que los estudios disponibles utilizaron criterios diferentes para definir este indicador (9) y anteriormente no predominaba el sistema de aseguramiento. Sin embargo, según datos del Ministerio de Salud, $25 \%$ de la población no contaba con cobertura de salud en 1991 (4), mientras que en 2003, durante la Encuesta Nacional de Calidad de Vida, 
se encontró que la proporción de la población sin seguro de salud era de $42 \%$ (10). De esta manera, aunque se suponía que la reforma de 1993 resolviera los problemas de inequidad del sistema de salud que afectaban a la población más pobre, $60 \%$ de las personas del estrato más pobre de la población no tenían seguro de salud en el año 2003, mientras que solo $10 \%$ de las personas del estrato socioeconómico alto no estaban aseguradas (10).

Uno de los aspectos que ha provocado mayor inequidad es el aumento del dinero que las familias invierten en la salud, también denominado gasto de bolsillo. De acuerdo con la Encuesta Nacional de Calidad de Vida de 2003, 40\% de las personas de los dos estratos más pobres que se sintieron enfermas y no consultaron un médico adujeron razones económicas (10), mientras que en la Encuesta Nacional realizada entre 1986 y 1989, este porcentaje fue de 28,5\% (9). En un estudio realizado entre 1985 y 1997 se encontró que aunque el gasto de bolsillo para la salud aumentó en todos los estratos sociales después de la reforma, el gasto subió en $90 \%$ en la población más pobre, mientras que en los tres décimos de mayores ingresos el aumento fue de $70 \%$ (11).

Sin embargo, tal vez el cambio más importante fue el deterioro de la red hospitalaria pública y de los programas de salud pública. La deuda de las empresas aseguradoras con los hospitales públicos ha llevado al cierre de ocho hospitales universitarios en los años de aplicación de la reforma (12). Igualmente se desarticuló la mayor parte de los programas de promoción de la salud y de prevención de enfermedades, por lo que aumentó la incidencia de las enfermedades prevenibles mediante vacunación. La cobertura de la vacunación infantil contra la difteria, la tos ferina, el tétanos, la tuberculosis, la poliomielitis y el sarampión se redujo de $68 \%$ en 1990 a $66 \%$ en 1995 y a $52 \%$ en 2000 (13).

La relación entre el gasto social y el producto interno bruto colombiano aumentó durante la década posterior a la adopción de la Constitución de 1991, pero empezó a disminuir a partir del año 2000 (14). El gasto social en la salud se comportó de manera similar. De acuerdo con un estudio realizado en 1997, el aumento del gasto social en la salud se debió a un mayor aporte de las familias a los gastos directos de salud (15). Esto representó un retroceso en la composición del gasto social, ya que los pobres gastaban en la salud un mayor porcentaje de sus ingresos. En los últimos años, el peso relativo del gasto directo en la salud realizado por las familias puede haber disminuido en relación con el total del gasto social en la salud, pero no hay estudios concluyentes al respecto $(16)^{3}$.

\footnotetext{
3 Las entidades gubernamentales autoras del informe de referencia aclararon que la información empleada estaba sujeta a revisión.
}

En Colombia, como en toda América Latina, el paradigma neoliberal-utilitarista sigue vigente después de 20 años de aplicación. Sin embargo, esto no se debe a su capacidad para resolver los problemas e inequidades en el ámbito de la salud, sino a la capacidad económica y coercitiva de sus agentes y beneficiarios, entre los que se encuentran las aseguradoras médicas, el Fondo Monetario Internacional y el Banco Mundial, así como una parte de los intelectuales y funcionarios públicos nacionales (17). Para los defensores del paradigma neoliberalutilitario, los problemas no se han generado por la fundamentación misma del paradigma, sino por una "aplicación inadecuada" que ha permitido conservar algunos de los elementos propios del sistema de salud anterior. Por lo tanto, su propuesta es continuar con la aplicación del modelo, pero de una forma más ortodoxa (18).

\section{El enfoque del derecho a la salud}

El derecho a la salud es el enfoque teórico y político que se ha contrapuesto a la hegemonía neoliberal-utilitarista. Por derecho a la salud se entiende el acceso a los servicios de promoción de la salud, de prevención y curación de enfermedades y de rehabilitación en los casos de deterioro de la salud. Esta categoría abarca también el acceso a una alimentación adecuada, a condiciones de trabajo sanas y seguras y a servicios de apoyo para el cuidado de la salud (19).

Como plataforma política, el derecho a la salud ha logrado aglutinar y movilizar diferentes fuerzas sociales en América Latina, particularmente en Colombia, unidas por el cuestionamiento de los fundamentos morales y de las consecuencias empíricas del paradigma neoliberal-utilitarista. Estas fuerzas sociales están constituidas por académicos y profesionales de la salud que trabajan en la llamada medicina social (20), además, por sindicalistas, líderes comunitarios, funcionarios de organizaciones no gubernamentales (ONG) y algunos empleados gubernamentales. El punto de convergencia de estas fuerzas sociales es el principio de que cualquier sistema de salud que pretenda resolver las inequidades de salud deberá partir de establecer una carta de derechos que no discrimine por ninguna condición social o económica. Para ello se requieren sistemas que contribuyan a superar la histórica fragmentación social imperante en América Latina y no a acentuarla.

Para contribuir a fundamentar el enfoque moral del derecho a la salud, que es el objetivo de este artículo, se deben responder las preguntas formuladas por Norman Daniels: ¿Qué tiene de especial el bien "salud" que justifique la aplicación de 
un principio de justicia en su distribución? ¿Por qué los mecanismos de asignación de los recursos para la atención sanitaria no se deben basar en el mercado? (19). Para argumentar las respuestas se seleccionaron algunos elementos éticos y morales provenientes de diversas escuelas de pensamiento.

\section{La teoría de la distribución igualitaria de los recursos}

Gosepath (21) elaboró una explicación muy didáctica sobre la diferencia entre la justificación legal y la justificación moral de los derechos sociales, como la salud y la educación, que resulta útil no solo para entender sus puntos de vista, sino para ubicar mejor este debate. Se entiende por "derecho" las demandas que unas personas - los portadores de los derechos- hacen a otras, justificadas o justificables mediante elementos jurídicos, ya sea por un deber contraído previamente, como un contrato, o por un principio moral, como el respeto igualitario.

Existen dos clases de derechos, los legales y los morales. Los derechos legales son pretensiones que se pueden reclamar judicialmente en un Estado y su violación es sancionada por los medios de coacción estatal. En otras palabras, los derechos legales son los respaldados por una ley positiva que los hace explícitos. El caso de los derechos morales es diferente, pues cada ser humano los tiene sin necesidad de que se cumplan condiciones especiales o una ley que los explicite. Para ejercer los derechos morales no se requiere ser ciudadano de un país determinado o pertenecer a una determinada comunidad. Estos derechos valen en virtud de la pertenencia a la comunidad humana. Son derechos universales.

Gosepath (21) propone basar los derechos sociales (educación, salud y trabajo, entre otros) en el mismo principio moral en que se fundamentan los derechos humanos, es decir, en el respeto igualitario que merecen todos los seres humanos. Este es el principio rector de todas las corrientes políticas de la sociedad moderna. Según Gosepath, del respeto igualitario se puede derivar una propuesta de distribución igualitaria de todos los bienes de que se disponga en la sociedad, lo que puede servir de fundamentación de los derechos sociales. Cuando todas las personas tienen interés en los bienes a distribuir - ya que todos deben ser respetados por igual por tener igual dignidad-, la satisfacción de las preferencias de todos será el principio más importante, siempre que no haya justificación para establecer una distribución desigual. No se exige una distribución igualitaria estricta en todos los casos, pero sí la necesidad de explicar con fundamentos razonables y universales los casos de distribución desigual. Sin embargo, cuando no haya razones de peso que justifiquen que la distribución no sea igualitaria, esta debe ser el punto de partida ineludible.

\section{La teoría de la igualdad de oportunidades}

En el campo de la salud, la teoría de la igualdad de oportunidades se deriva de la interpretación que Daniels (19) hizo de la teoría de la justicia de Rawls (22). Según Rawls, es necesario aplicar principios de justicia al funcionamiento de la estructura básica de la sociedad, es decir, de las principales instituciones políticas, sociales y económicas y de la manera como ellas interactúan para formar un sistema de cooperación social. En términos generales, se trata de la constitución política, la organización del gobierno, la estructura y las reglas que rigen los mercados, y las formas de propiedad legalmente reconocidas (22).

Daniels (19) plantea que la política de salud - como parte de la estructura básica de la sociedad - también se debe regir por principios de justicia, ya que genera condiciones que afectan a la realización de los proyectos de vida de las personas. La política sanitaria no se puede basar en el mercado, porque quienes no logren participar de él no tendrían acceso a la satisfacción de las necesidades de salud y podrían sufrir daños -en algunos casos irreversibles- que limitarían el desarrollo de sus proyectos de vida y en ocasiones los de sus descendientes. Una sociedad organizada exclusivamente según las normas del mercado no logrará legitimidad ante los ojos de sus ciudadanos y estos no se pueden considerar a sí mismos personas libres e iguales ni se sentirán comprometidos activamente con su futuro.

Según Rawls (23), uno de los principios que debe regir la estructura básica de la sociedad es el de la justa igualdad de oportunidades. Esto significa que la posibilidad de seleccionar una profesión o de ocupar un cargo que implique ventajas sociales o económicas no puede depender de las contingencias sociales y económicas de las personas. Es decir, no se puede permitir que la igualdad de oportunidades sea solamente formal y que los cargos y las profesiones se abran solo a quienes tengan capacidad y deseo de ocuparlas, pues esto acarrearía desigualdades debido a la capacidad innata y al nivel de preparación que las condiciones sociales y económicas de las personas hayan permitido, lo que desde el punto de vista moral es arbitrario.

De lo que se trata, según Rawls, es de instaurar una serie de instituciones sociales que contribuyan a derrumbar las barreras de clase que convierten los cargos y las prerrogativas en privilegios de unos pocos. Aunque Rawls no define de manera detallada qué instituciones se requieren para garanti- 
zar la igualdad de oportunidades, Daniels estructura su argumentación y propone que el acceso a los servicios de salud sea parte de ese conjunto de instituciones (20). Si una persona tiene un problema de salud y la sociedad no le ofrece los servicios necesarios para recuperarse y rehabilitarse, esa persona tendrá menos oportunidades que otras.

\section{La teoría del desarrollo como libertad}

En muchos de sus escritos, Sen propone reconsiderar los conceptos de desarrollo y de libertad (24). Para este autor, la libertad tiene dos facetas: la de proceso y la de oportunidad. La primera se refiere a la elección autónoma, a la capacidad de las personas de tener en sus manos los mecanismos de control. Se refiere al procedimiento de cada persona de decidir libremente por sí misma. La faceta de oportunidad, en cambio, se refiere a la libertad de lograr los objetivos o cosas que cada uno valora, es decir, está relacionada con las oportunidades reales que la sociedad brinda a las personas para conseguir lo que les es importante.

Sen (25) afirma que la libertad no puede ser entendida únicamente como una lista de derechos liberales negativos que privilegian la libertad de proceso, sino que la noción de libertad debe ampliarse de manera que abarque la faceta de oportunidad. El autor argumenta que no son aceptables los supuestos liberales de la prioridad de ciertos derechos, pues el respeto de los derechos liberales de algunos - como el derecho a la propiedad-puede llevar a someter a los más pobres a catástrofes sociales - como las hambrunas generalizadas y la falta de asistencia médica- y estas situaciones no tienen respaldo ético alguno.

Considerar la faceta de oportunidad de la libertad junto a la de proceso implica, según Sen, que el estado social se mida según el grado de libertad de que disfrutan sus habitantes para lograr su bienestar. Este grado de libertad está dado por la posibilidad que tienen las personas de escoger entre diversas alternativas valiosas para elaborar sus proyectos de vida.

Al aplicar la teoría de Sen a la evaluación de la política de salud desarrollada por un Estado, no solo se deben tener en cuenta las metas alcanzadas por las personas - como su estado nutricional o de salud-, sino la posibilidad real que tuvieron de escoger otras alternativas más valiosas que las alcanzadas.

La libertad, desde el punto de vista que propone Sen, significa no tener obstáculos para aprovechar las oportunidades. En el caso de la salud y de la nutrición, se trata de alcanzar un estado sanitario adecuado y de no sufrir los efectos negativos que podrían provocar el deterioro de la salud y la mala nutrición, ya que pueden limitar la capacidad de llevar la vida valiosa a la que se aspira (26).

El objetivo de brindarles a todos los ciudadanos la oportunidad de alcanzar un estado adecuado de salud sin enfermedades evitables, así como la oportunidad de estar suficientemente bien nutrido, deben formar parte de los objetivos de desarrollo de toda sociedad.

\section{La teoría de la conexión con el derecho a la vida}

Otro punto de vista desde el cual se puede fundamentar moralmente el derecho a la salud es el de su conexión con el derecho a la vida. A esta consideración han apelado millares de colombianos que han visto cómo el nuevo sistema de salud les niega el acceso a los servicios y pone en riesgo su supervivencia (2). El planteamiento central es que cuando se niega el derecho a la salud se pone en riesgo el derecho a la vida, que es el derecho fundamental por excelencia según la Constitución. Esta teoría se ha convertido en una de las pocas vías de intervención estatal en el funcionamiento del mercado de la salud en Colombia. Mediante el mecanismo de la tutela (denominado recurso de amparo en otros países) se ha sustentado la conexión de los dos derechos - a la vida y a la salud-y en muchos casos, los jueces han logrado romper los mecanismos de exclusión creados por el nuevo sistema de salud. Pero esta teoría tiene limitaciones, pues como la tutela es individual, no constituye una regla aplicable en casos similares y solo se puede emplear cuando la negación del derecho a la salud amenaza la vida de las personas, condición que no siempre se presenta o que no siempre se puede demostrar.

\section{Críticas a la fundamentación moral del derecho a la salud}

Cuando se plantea la posibilidad de considerar a la salud un derecho fundamental, la primera crítica surge en la orilla liberal, pues como se mencionó anteriormente, los teóricos liberales consideran que los únicos derechos fundamentales son los civiles y los políticos. Es precisamente para responder a esta crítica que se ha expuesto cómo el derecho a la salud y los derechos sociales en general tienen un fundamento moral.

Por otra parte, los teóricos liberales sostienen que es imposible definir y reglamentar de manera específica el contenido concreto del derecho a la salud y que los costos de aplicar esa definición en sociedades que enfrentan crecientes demanda y escasez de recursos serían muy elevados. Para responder a esas críticas, muchos autores argumentan 
que el Estado y la sociedad también incurren en grandes costos para aplicar la definición técnica de los derechos negativos (21) y, sin embargo, se han hecho grandes esfuerzos para incorporarlos en la constitución de la mayoría de los países y se han creado numerosas entidades gubernamentales y de la sociedad civil encargadas de velar por su cumplimiento, como las instituciones establecidas para proteger el derecho al voto, a la libertad religiosa o a la propiedad privada.

Otra crítica a la teoría de los derechos en general -no específicamente a los derechos sociales-es la formulada por algunos teóricos de la escuela comunitarista, para quienes la teoría de los derechos contiene sesgos por estar basada en derechos que se exigen y se disfrutan de manera individual. Según ellos, el considerar que los seres humanos están interesados solamente en sus propios planes de vida, sin valores comunitarios, constituye una imagen muy vaga de la condición humana (27). Para algunos comunitaristas, el problema radica en que - según la teoría de los derechos individuales- las sociedades están conformadas por personas cuya identidad está dada de manera independiente de su relación con los otros.

Según Sandel, la teoría de los derechos individuales considera a los seres humanos ante todo como individuos y después como seres que viven en sociedad y por ello en las sociedades modernas priman los derechos individuales sobre los colectivos (27). Para los comunitaristas, estos criterios suponen el desinterés mutuo entre los ciudadanos y el predominio de un sentimiento individualista que rechaza otras motivaciones, como la benevolencia, el altruismo y los sentimientos comunitarios.

En el presente artículo no se pretende formular una respuesta exhaustiva a la crítica comunitarista, pero se debe subrayar que desde un punto de vista práctico, en los sistemas de salud estructurados según los conceptos comunitaristas - del bien común - no se excluye la posibilidad de considerar a cada ciudadano como titular del derecho a la salud, dadas las múltiples relaciones existentes entre lo individual y lo colectivo que condicionan la situación de salud en cualquier sociedad.

En síntesis, si bien según los conceptos liberales, solamente los derechos civiles y políticos se consideran fundamentales y se interpreta la libertad solo como la libertad de proceso y la ausencia de mecanismos de coerción, hay argumentos éticos y morales que apoyan el criterio de que la salud es un derecho humano fundamental. En el presente artículo se han analizado tres elementos del pensamiento ético-moral: la justa igualdad de oportunidades, el respeto igualitario que merecen las personas -lo que exige la distribución igualitaria de los bienes y de las responsabilidades sociales, siempre que la distribución desigual no pueda justificarse- y la libertad, entendida como la oportunidad de cada persona de realizar un proyecto de vida escogido entre alternativas socialmente valiosas.

Agradecimientos. La investigación en que se basa este artículo se realizó con el apoyo financiero de la Universidad de Antioquia, en particular de su Escuela de Nutrición y Dietética. La autora agradece el asesoramiento académico del profesor Francisco Cortés, del Instituto de Filosofía, y de Jorge Bernal, Director de la Corporación Región de Medellín.

\section{SYNOPSIS}

\section{The right to health in Colombia: a proposal for} laying down its moral foundations

The health system reform that was carried out in Colombia in 1993 was based on neoliberal doctrine. That reform increased the inequity in the allocation of resources, access to health services, and the distribution of spending on health. Societal organizations, academic groups, health workers, and some members of the political sector in Colombia have opposed the neoliberal reform, and they have supported the right to health as an alternative for dealing with the inequities in the health sector. According to liberal theories, only civil and political rights are fundamental, and liberty is assumed to be only the freedom of process and the absence of coercive mechanisms. However, there are arguments that support the viewpoint that health is a fundamental human right. This piece analyzes some ethical and moral principles that can help establish the moral foundations for the right to health. Among these principles are equality of opportunity, that part of being free is for people to have the right to develop themselves as human beings, that the right to health is connected to the right to life, that all people deserve equal respect (which requires the equal distribution of goods and of societal responsibilities), and that liberty is the opportunity that all persons should have to carry out the plans that they have for their lives, as chosen from a variety of socially useful alternatives.

Key words: social justice, delivery of health care, health resources, resource allocation, human rights, health care reform, Colombia. 


\section{REFERENCIAS}

1. Aday L. An expanded conceptual framework of equity: implications for assessing health policy. En: Albrecht GL, Fitzpatrick R, Scrimshaw SC, eds. Handbook of social studies in health and medicine. Thousand Oaks (California): SAGE Publications; 2000. Pp. 481-92.

2. Defensoría del Pueblo, ProSeDher. E derecho a la salud en la Constitución, la jurisprudencia y los instrumentos internacionales. Santa Fe de Bogotá: Defensoría del Pueblo, ProSeDher; 2003.

3. World Health Organization. The world health report. Health systems: improving performance. Geneva: WHO; 2000.

4. Colombia, Ministerio de Salud. Diagnóstico de la salud en Colombia. En: Crisis y perspectivas de la salud en Colombia. Medellín: Universidad de Antioquia; 1991. Pp. 43-68.

5. Frenk J. Hacia la reforma del sistema de salud. México, D.F.: Fundación Mexicana para la Salud; 1994.

6. Gracia D. Fundamentos de bioética. Madrid: Eudema; 1989.

7. Morrow R, Byant J. Health policy approaches to measuring and valuing life: conceptual and ethics issues. Am J Public Health. 1995;85:1356-60.

8. Whitehead M. The concepts and principles of equity and health. Copenhagen: WHO Regional Office for Europe; 1985. Hallado en: http://www.who.dk/ Document/PAE/conceptsrpd414.pdf Acceso el 15 de junio de 2005.

9. Echeverri ME. Estudios de accesibilidad a los estudios de salud en Colombia antes y después de la Ley 100/93. En: La salud al derecho. Medellín: Universidad de Antioquia, Movimiento Nacional por la Salud y la Seguridad Social, Gobernación de Antioquia; 2001. Pp. 75-120.
10. Colombia, Departamento Administrativo Nacional de Estadísticas. Encuesta Nacional de Calidad de Vida 2003. Salud Colombia [publicación periódica en línea] 2004; enero-marzo [citada 2004 noviembre 4]; 74. Hallado en: http:// www.salud colombia.com/actual/ salud74/ENCV2003a_archivos/frame. htm

11. Castaño RA, Arbelaez JJ, Giedon U, Morales LG. Equitable financing out of pocket payments and the role of health care reform in Colombia. Health Policy Plan. 2002;17(90001):5-11. Hallado en http://heapol.oupjournals.org/cgi/ content/abstract/17/suppl_1/5. Acceso el 15 de junio de 2005.

12. Paredes N. La salud pública: entierro definitivo. En: Borrero C, ed. Reelección: el embrujo continúa. Santa Fe de Bogotá Plataforma Colombiana de Derechos Económicos, Sociales y Culturales; 2004. Pp. 109-18.

13. Profamilia. Salud sexual y reproductiva en Colombia. Encuesta Nacional de Demografía y Salud 2000. Santa Fe de Bogotá: Profamilia; 2000.

14. Sarmiento L. Malestar social y política pública. En: Borrero C, ed. Reelección: el embrujo continúa. Santa Fe de Bogotá: Plataforma Colombiana de Derechos Económicos, Sociales y Culturales; 2004. Pp. 89-98.

15. Departamento Nacional de Planeación. Coyuntura económica e indicadores sociales. Santa Fe de Bogotá: Departamento Nacional de Planeación; 2000.

16. República de Colombia, Departamento Nacional de Planeación, Ministerio de Protección Social. Proyecto cuentas de salud. Salud Colombia [publicación periódica en línea] 2004; abril-junio [citada
2004 oct 8]. Hallado en: http://www. saludcolombia.com/actual/salud75/ estad75.htm

17. Almeida C. Reforma de sistemas de servicios de salud y equidad en América Latina y el Caribe: algunas lecciones de los años 80 y 90. Cad Saude Publica. 2002;18:905-25.

18. Fedesarrollo. El sistema de seguridad social en salud, logros y retos. Coyuntura Social (Santa Fe de Bogotá). 2000;2: 32-53.

19. Daniels N. Justice health care. Cambridge (U.K.): Cambridge University Press; 1985.

20. Tajer D. Latin American social medicine: roots, development during the 1990s and current challenges. Am J Public Health. 2003;93:2023-7.

21. Gosepath S. Consideraciones sobre las fundamentaciones de los derechos humanos sociales. En: Alonso MA, Giraldo J, eds. Ciudadanía y derechos humanos sociales. Medellín: Escuela Nacional Sindical; 2001. Pp.15-58.

22. Rawls J. Teoría de la justicia. México, D.F.: Fondo de Cultura Económica; 1997.

23. Rawls J. Liberalismo político. Santa Fe de Bogotá: Fondo de Cultura Económica; 1996.

24. Sen A. Bienestar, justicia y mercado. Barcelona: Paidós, Universidad Autónoma de Barcelona; 1997.

25. Sen A. Desarrollo y libertad. Santa Fe de Bogotá: Planeta; 2000.

26. Sen A, Nusbaumm M. La calidad de vida. México, D.F.: Fondo de Cultura Económica; 1996.

27. Sandel W. El liberalismo y los límites de la justicia. Barcelona: GEDISA; 2002. 